\title{
LITURGIA DELLA ECCLESIA E LITURGIA DEL MONASTERO NELLA TRADIZIONE AMBROSIANA
}

\section{CESARE ALZATI}

UDC: $27-788(450.251) " 04 / 10 "$ 27-28

Original scientific paper

Manuscript received: 24. 12. 2016.

Revised manuscript accepted: 07. 01. 2017.

DOI: 10.1484/J.HAM.5.113704
C. Alzati

Academia Romena

Bucarest, Romania

Milano nei secoli alto medioevali ha conosciuto una singolare pluralità di fondazioni monastiche urbane, la cui vita cultuale si sviluppava nel quadro della tradizione rituale ambrosiana. Il paradigma di tale tradizione era rappresentato dalle solenni celebrazioni proprie dell'arcivescovo e dei cardinali della Chiesa milanese; in contesto monastico veniva riproposta una specifica declinazione di tale paradigma, adattata alla realtà del cenobio. Di tali comunità monastiche ambrosiane ci sono pervenute in particolare due significative testimonianze manoscritte: il Messale di Armio e il Messale di S. Simpliciano, al quale va aggiunto il messale palinsesto del ms. Harleian 2510 della British Library. Fuori Milano la prassi liturgica ambrosiano-monastica ha conosciuto nel XIV secolo una singolare fortuna nella Praga di Carlo IV, dove un monastero di rito ambrosiano fu dall'imperatore affiancato al cenobio glagolitico, da lui precedentemente istituito. Se le guerre hussite misero fine a tale presenza ambrosiana sulle rive della Vltava, a Milano, con l'affermarsi dei Mendicanti e della loro esenzione, vennero progressivamente meno le condizioni canoniche e istituzionali, che nei secoli alto medioevali avevano permesso quell'organica interazione tra le diverse componenti della Chiesa ambrosiana, interazione, efficacemente manifestata anche nei libri di culto.

Keywords: Ambrosian liturgy, Monastic liturgy, Charles IV emperor, Prague monasteries

Nella ricerca storica in merito alla formazione e all'evoluzione della preghiera con cui le Chiese cristiane tradizionalmente hanno accompagnato il volgere del giorno, scandendone le fasi con la propria adorazione e la propria lode, Anton Baumstark ha richiamato con forza l'attenzione sulle due distinte matrici, da cui il patrimonio rituale in questione è venuto sviluppandosi. Si tratta della forma di celebrazione, ch'egli chiama «rite cathédral», e delle modalità di preghiera proprie delle comunità monastiche, modalità da lui identificate come «rite monastique» ${ }^{1}$. In realtà, per la prima di tali forme rituali, lo stesso Baumstark in Nocturna laus ritenne di dover ricorrere alla definizione, a mio giudizio più consona, di «Gemeindefeier» $»^{2}$. Per identificare quella stessa tipologia celebrativa, in tempi a noi più vicini, George Guiver, considerando chi vi prendeva parte, ha usato la definizione di «people's office».

\section{«RITE CATHÉDRAL»: UNA DEFINIZIONE PROBLEMATICA}

La validità e fecondità della distinzione tipologica, evidenziata dal Baumstark e universalmente recepita, non cancella la problematicità dell'espressione «rite cathédral», che grande fortuna ha conosciuto a partire dalla pubblicazione di Liturgie comparée. In effetti non si può non rimarcare come il concetto di «cattedrale», quale fino a oggi sostanzialmente si perpetua nelle Chiese europee con le sue implicazioni istituzionali e canoniche, sia venuto definendosi - in Occidente - nell'avanzata età medioevale. Le affermazioni di Mauro Ronzani al riguardo mi appaiono pienamente condivisibili ${ }^{4}$, anche se va precisato che l'espressione «ecclesia cathedralis» è reperibile già nell'anno 666 nei testi del concilio provinciale di Mérida (segnatamente nei cann. 10 e 12$)^{5}$. Stante questa connotazione occidentale e relativamente tarda della realtà istituzionale attualmente identificata dal termine «cattedrale», l'applicazione del termine stesso alla situazione ecclesiale antica appare non poco problematica.

In effetti in età patristica, nelle città (escludendo le megalopoli Roma e Alessandria), l'unicità di celebrazione (ossia la convergenza dell'intera comunità urbana là dove il vescovo celebrava circondato dal suo clero) era principio rigoroso, ossia sussisteva anche qualora la comunità disponesse di una pluralità di edifici di culto. Questa unicità di celebrazione era la concretizzazione delle idealità, che un tempo (in situazioni peraltro alquanto diverse) avevano ispirato le parole dirette da Ignazio d'Antiochia ai fratelli di Filadelfia: «Abbiate cura d'essere tutti partecipi di un'unica eucaristia. Una è la Carne del nostro Signore Gesù Cristo, e uno è il calice nell'unione del suo Sangue; uno è l'altare come uno solo il vescovo con il presbiterio e i diaconi» ${ }^{6}$.

\footnotetext{
${ }^{1}$ Cfr. A. BAUMSTARK, Liturgie comparée, Monastère d'Amay, Chevetogne, $1953^{3}\left(1932^{1}\right)$, p. 118 ss.

${ }^{2}$ ID., Nocturna laus. Typen frühchristlicher Vigilienfeier und ihr Fortleben vor allem im römischen und monastischen Ritus, cur. O. Heiming, Münster, 1956 (Litutgiewissenschaftliche Quellen und Forschungen, XXXII).

3 G. GUIVER, Company of voices: Daily Prayer and the People of God, London, 1988, p. 52-53.

${ }^{4}$ M. RONZANI, 'Chiesa del Comune', 'cattedrale civica', 'Stadtstift': S. Petronio e un possibile capitolo di storia comparata della Chiesa cittadina nel basso medioevo, in Una basilica per una città. Sei secoli in San Petronio, Atti del convegno di studi per il sesto centenario di fondazione della basilica di San Petronio (1390-1990), curr. M. Fanti - D. Lenzi, Bologna, 1994 (Istituto per la storia della Chiesa di Bologna. Saggi e ricerche, V), p. 35-50.

${ }_{5}$ Concilios Visigóticos e Hispano-Romanos, ed. J. Vives (- T. Marín Martínez - G. Martínez Díez), Barcelona-Madrid, 1963, p. $332-334$.

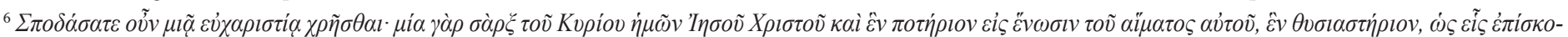

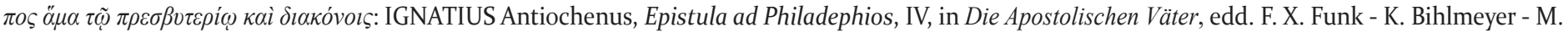
Whittaker, germ. vertt. M. Dibelius - D. A. Koch, vers. revv. A. Lindemann - H. Paulsen, Tübingen, 1992, p. 220. Cfr. anche Epistula ad Magnesios, VII, 1, 2,
} 
Nel contesto della prassi qui delineata diviene pienamente comprensibile perché tanto radicale sia stata nel IV-V secolo (ma non solo) l'opposizione dei vescovi al fatto che in città le comunità scismatiche godessero di propri luoghi cultuali ${ }^{7}$.

Oltre all'unicità della celebrazione, ulteriore aspetto fondamentale della prassi celebrativa cristiana in età patristica fu il culto stazionale, cui John Baldovin ha dedicato un volume ormai classico ${ }^{8}$. Nei centri urbani dotati di sede episcopale, il luogo di celebrazione del vescovo poteva, in dipendenza delle feste, traslarsi nei diversi edifici di culto della città, dove ci si recava per compiervi la statio. Anche in tale caso si trattava, pertanto, di celebrazione unitaria presieduta dal vescovo e per la quale si raccoglieva l'intera Chiesa, ossia il clero (almeno nelle sue componenti più rappresentative) e il popolo9. Alla luce di questo aspetto 'episcopale' (oltre che unitario) proprio delle celebrazioni stazionali, l'espressione «chiesa episcopale» per designare l'edificio delle abituali celebrazioni presiedute dal vescovo (espressione proposta dal Ronzani e che io pure ho utilizzato) appare non sufficientemente connotativa per la realtà, che intende definire ${ }^{10}$.

In ogni caso, la non idoneità del ricorso al termine «cattedrale» diviene ancor più palese in rapporto all'Oriente. Nel cremlino di Mosca si trova una pluralità di edifici di culto che godono del titolo di «co6op / psl.: sßborß». Esso viene abitualmente (e impropriamente) tradotto in lingue occidentali con «cattedrale». Ė significativo il fatto che il mondo romeno, ortodosso ma di lingua neolatina, abbia anch'esso conosciuto il termine «sobor» ma, per esprimerne il concetto, si trovi sprovvisto di un adeguato termine di radice latina e attualmente ricorra al neologismo catedrală, che ha determinato un generale (anche se non assoluto) restringimento nell'uso del termine stesso, secondo la sua accezione occidentale. In realtà il russo «собор» ripropone

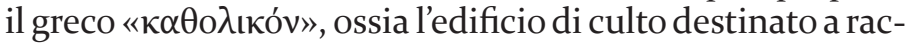

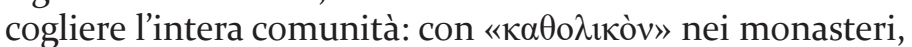

a cominciare dall'Athos, si identifica la grande chiesa, dove convergono i monaci dei cenobi per l'officiatura antelucana e vespertina. Siamo ben lontani dal significato corrente di «cattedrale».

Si tratta di una questione lessicale, ma in realtà è anzitutto una questione concettuale, importante a livello storiografico, ma non meno rilevante a livello ecclesiologico. Al riguardo può essere interessante considerare la terminologia utilizzata in una sinodo della sede episcopale di Auxerre alla fine del secolo VII. Il vescovo Tetricius nel primo anno del suo episcopato volle garantire una solenne officiatura quotidiana in quella che il citato concilio di Mérida chiama pure «ecclesia principalis $»^{11}$, ossia l'edificio di culto dove abitualmente egli esercitava il proprio ministero episcopale; per questo impose al clero delle varie istituzioni ecclesiastiche cittadine e rurali di assolvere a turno il compito di assicurare la solennità della celebrazione. Nell'impartire tali disposizioni, egli parla della «aecclesia sancti Stephani», dicendo che a essa devono recarsi i chierici della varie «basilicae» urbane (identificate dal nome dei santi titolari), nonché le comunità monastiche e il clero rurale (designato mediante i toponimi dei rispettivi villaggi) ${ }^{12}$.

«Ecclesia» è dunque il termine con cui Tetricius identifica l'edificio di culto del complesso episcopale, distinguendolo dagli altri analoghi edifici presenti nel territorio urbano, designati col termine di «basilicae». «Ecclesia», ossia luogo di manifestazione della natura profonda della comunità cristiana, che nella celebrazione dei Divini Misteri (la mensa eucaristica, il lavacro battesimale, la crismazione, l'unzione dei malati, il perdono concesso ai penitenti, la liberazione dai demoni) vedeva l'edificio, in cui era raccolta, trasfigurarsi nella rutilante porta del Cielo, come canta l'inno ambrosiano in dedicatione ecclesiae maioris ${ }^{13}$.

Sicché, alla luce della terminologia del vescovo Tetricius e tenendo conto delle considerazioni di George Guiver, mi è parso opportuno designare la forma rituale, che cor-

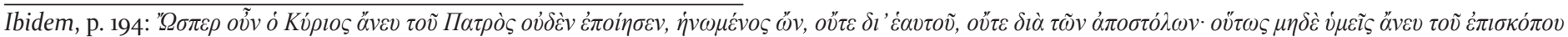

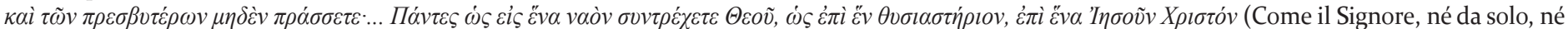
insieme agli apostoli, nulla ha fatto separatamente dal Padre, col quale è una cosa sola, così voi non fate nulla senza il vescovo e i presbiteri ... Accorrete dunque tutti come all'unico Tempio di Dio, verso l'unico altare, verso l'unico Gesù Cristo).

7 Alla unicità di celebrazione facevano eccezione - come si è segnalato - le grandi megalopoli, che assai per tempo conobbero una pluralità di poli cultuali. A Roma, in tale situazione, il segno dell'unità diveniva il fermentum, ossia la particola eucaristica, che il papa inviava ai preti celebranti nei tituli, perchévenisse immessa nel loro calice. La medesima prassi sembra essere stata ripresa anche da alcuni vescovi dell'Italia Suburbicaria (con dissenso del loro metropolita) in riferimento ai preti da loro dipendenti e insediati nei territori rurali: INNOCENTIUS I Romanus, Epistula ad Decentium Eugubinum, ed. R. Cabié, La lettre du Pape Innocent I I à Décentius de Gubbio (19 mars 416), Louvain, 1973 (Bibliothèque de la Revue d'Histoire Ecclésiastique, 58), p. 26. 92 - 28. 101.

Con la crescita delle comunità, a Roma si iniziò a iterare la celebrazione nella medesima chiesa, suscitando alla metà del V secolo lo stupore del papa di Alessandria, Dioscoro, che ne chiese ragione al collega romano Leone: LEO I Romanus, Epistula IX, 2: in Patrologiae Cursus Completus. Series Latina, Migne, Parisiis, 1878 ss. [= PL], 54, c. 627: «Necesse est autem, ut quaedam pars populi sua devotione privetur, si unius tantum Missae more servato, sacrificium offerre non possint, nisi qui prima diei parte convenerint».

${ }^{8}$ J. F. BALDOVIN, The Urban Character of Christian Worship: the Origins, Development and Meaning of Stational Liturgy, Roma, 1987 (Orientalia Christiana Analecta, 228).

${ }_{9}$ Ciò avveniva, in occasione delle stationes, anche nelle megalopoli, come indica verso al fine del VII secolo l'Ordo I romano: M. ANDRIEU, Les Ordines romani du haut Moyen-Age, II, Louvain, 1948 [Spicilegium Sacrum Lovaniense, 23], p. 67 ss.

${ }^{10}$ Diverso è il caso della definizione «complesso episcopale», usata in ambito archeologico, che riesce ad identificare compiutamente l'insieme designato, in cui - oltre a chiesa/e - convergono pure battistero/i e domus episcopi.

"Can. 12: Concilios Visigóticos e Hispano-Romanos, ed. J. Vives, p. 333.

${ }^{12}$ Concilia Galliae. A. 511 - A. 695, ed. Ch. de Clercq, Turnholti, 1963 (Corpus Christianorum. Series Latina [= CCL], 148/A), p. 323-326 (Synodus dioecesana Autissiodorensis. 692-696).

${ }^{13}$ Il testo dell'inno può vedersi in Manuale Ambrosianum ex codice saec. XI olim in usum canonicae Vallis Travaliae, ed. M. Magistretti, II, Mediolani, 1904, p. 359-36o (datazione di questo codice al XII secolo ineunte è stata proposta da O. HEIMING, Die ältesten ungedruckten Kalender der mailändischen Kirche, in Colligere fragmenta. Festschrift Alban Dold, Erzabtei Beuron, 1952, p. 214-215); cfr. anche: Gli Inni del Breviario Ambrosiano, ed. E. Garbagnati, vert. et 
rentemente tra i liturgisti si designa come rite cathédral, facendo riferimento al suo carattere di «rito della ecclesia»: definizione che congiuntamente allude sia alla Ecclesia che tale rito celebra, sia alla ecclesia in cui abitualmente tale rito si celebra.

\section{TRA ECCLESIA E CENOBIO URBANO: LE INTERAZIONI IN AMBITO MILANESE}

Nel suo fondamentale profilo storico della evoluzione conosciuta dall'officiatura quotidiana nelle diverse tradizioni cristiane, Robert Taft ha giustamente rimarcato il fenomeno, sviluppatosi a partire dal IV secolo avanzato, dei monasteri di tipo urbano e alla peculiare interazione che in essi si è realizzata tra il modello della celebrazione ecclesiale urbana e gli usi monastici ${ }^{14}$. Quest'ultimo fenomeno risulta particolarmente rilevante in riferimento all'analisi dei libri di culto monastici d'ambito ambrosiano. Essi, infatti, si legano a monasteri maschili sorti nell'immediato suburbio (tali insediamenti erano ben sei nella Milano altomedioevale) ${ }^{15} \mathrm{e}$ ripropongono - quantunque in forme legate al loro specifico cronotipo - una dinamica analoga a quella che caratterizzò la declinazione del modello celebrativo ecclesiale nell'ambito delle comunità di asceti connesse ai centri urbani in Oriente.

Quale fosse nell'ultima fase della tarda antichità l'autorevolezza del modello celebrativo ecclesiale ambrosiano, anche ai fini della sua recezione in ambito monastico, è emblema- ticamente mostrato dall'Antifonario di Bangor, che - con riferimento alle lodi matutine - riflette, all'interno di una comunità monastica irlandese, l'ordinamento rituale tipico di Milano ${ }^{16}$. Ma se vi fu influsso dell'officiatura ecclesiale milanese sulle comunità monastiche, anche non milanesi, è pur vero che gli usi monastici non mancarono d'influire a loro volta sull'officiatura, quale venne stabilizzandosi a Milano. In effetti, la celebrazione antelucana, se nei Sabati e nelle Domeniche presenta un tipico andamento ecclesiale (nelle Domeniche con evidente affinità rispetto al modello gerosolimitano attestato da Egeria, caratterizzato dalla recita di tre soli «psalmi») ${ }^{17}$, nelle feriae appare chiaramente ispirata al modello monastico: proclamazione dei Salmi in lectio continua ${ }^{18}$.

È comunque sintomatico che l'Ordo arcivescovile-cardinalizio del cicendelarius Beroldo, databile ad anno di poco successivo al 1126 (ma nel quale trovano conferma anche usi rituali ben più antichi, e perfino tardo antichi), delinei un'officiatura giornaliera, in cui per la sola Quaresima viene segnalato la celebrazione della Ore minori ${ }^{19}$.

\section{LIBRI PER LA CELEBRAZIONE EUCARISTICA NEI MONASTERI URBANI MILANESI}

Se la struttura dell'officiatura quotidiana in ambito ambrosiano ha conservato attraverso i secoli alcuni elementi tardo antichi che, spesso radicati nel modello gerosolimita-

adn. L. P. Colombo, Milano, 1897, pp. 96-98. Un ampio commento ai contenuti dell'inno in questione è stato sviluppato da P: BORELLA, La dottrina canonico-liturgica dell'inno "Christe cunctorum". Sermone, in Synodus Mediolanensis XLII. In metropolitana ecclesia diebus VIII, IX et X Octobris anno Domini MCMXXXV celebrata, Mediolani 1936, p. 131-157; inoltre, C. ALZATI, Pane, memoria, mistero nelle tradizioni ecclesiali dell'Italia Settentrionale, «Brixia sacra. Memorie storiche della diocesi di Brescia», terza serie, XXI, 1-4 (2016), p. 5-21.

${ }^{14}$ Cfr. R. TAFT, La Liturgia delle Ore in Oriente e in Occidente, (Cinisello Balsamo, 1988') Roma, 2001² , p. 105 ss. [trad. it., con marginali interventi, di The Liturgy of the Hours in East and West, Collegeville (U.S.A.), (1986') $1993^{2}$, p. 75 ss.].

${ }^{15} \mathrm{~A}$ loro volta, la comunità monastiche femminili assommavano a sette. Prescindendo dalla specifica (e inconfrontabile) situazione romana, tale concentrazione di monasteri urbani a Milano appare, nel contesto occidentale, assolutamente insolita. Al riguardo si rinvia a G. PICASSO, Monasteri e città a Milano in età comunale, in Atti dell'11 ${ }^{\circ}$ Congresso internazionale di studi sull'Alto Medioevo. Milano, 26-30 ottobre 1987, I, Spoleto, 1989, p. 375-393.

${ }^{16}$ Ed. F. E. WARREN, The Antiphonary of Bangor: An Early Irish Manuscript in the Ambrosian Library at Milan, London, 1893-1895 (Henry Bradshaw Society, 4-5). Per le consonanze con la celebrazione ecclesiale matutina milanese: M. CURRAN, The Antiphonary of Bangor and the Early Irish Monastic Liturgy, Dublin, 1984.

${ }^{17}$ EGERIA, Itinerarium, XXIV, 9, ed. P. Maraval, Paris, 2002² (Sources Chrétiennes [= SCh], 296), p. 242-244; per l'ambito ambrosiano: Psalterium, in Manuale Ambrosianum ex codice saec. XI olim in usum canonicae Vallis Travaliae, ed. M. Magistretti, I, Mediolani, 1905, p. 165-170; cfr. E. CATTANEO, Il Breviario Ambrosiano, Milano, 1943, p. 167; C. ALZATI, Il salmo e l'incenso. Antiche radici e nuovi sviluppi nell'officiatura ecclesiale, in ID., Il Lezionario della Chiesa Ambrosiana. La tradizione liturgica e il rinnovato «ordo lectionum», Città del Vaticano - Milano, 2009, p. 107-109, 112-114. Particolarmente significativa appare l'impronta gerosolimitana presente nelle officiature antelucane di quello che già in Ambrogio troviamo identificato come il Triduum Sacrum (AMBROSIUS, Epistula e. c. XIII (Maur.: XXIII): Dominis fratribus dilectissimis episcopis per Aemiliam constitutis, 13, ed. M. Zelzer, Vindobonae, 1982 [Corpus Scriptorum Ecclesiasticorum Latinorum (= CSEL), 82, 3), pp. 227-228: «Cum igitur Triduum illud Sacrum in ebdomadam proxime concurrat ultimam, intra quod Triduum et passus est et quievit et resurrexit, de quo Triduo ait: "Solvite hoc templum et in triduo resuscitabo illud"»). In tali giorni gli echi gerosolimitani sono rintracciabili nella lettura delle Passiones nella notte che apre alla Feria VI in Parasceve (A. RENOUX, Le codex Arménien Jérusalem 121, I: Introduction. Aux origines de la liturgie hiérosolimitaine. Lumières nouvelles, Turnhout, 1969 [Patrologia Orientalis, 35/1, n 163], p. 147-155; per l'ambito ambrosiano: BEROLDUS, Ordo et caeremoniae ecclesiae Ambrosianae Mediolanensis (= BEROLDUS), ed. M. Magistretti, Mediolani, 1894, p. 39-40; cfr. C. ALZATI, «Solemnitatum omnium honoranda solemnitas». La Chiesa Ambrosiana e il Mistero Pasquale, in ID., Il Lezionario della Chiesa Ambrosiana [cit. nella presente nota], p. 324-325); nonché nell'esclusivo carattere salmodico della preghiera nella notte susseguente alla "sottrazione dello Sposo" (EGERIA, Itinerarium, XXXVII, 9, SCh, 296, p. 290; analogamente il Lezionario armeno non prevedeva alcuna forma strutturata di celebrazione: A. Renoux, Le codex Arménien Jérusalem 121, II: Édition comparée du texte et de deux autres manuscrits, Turnhout, 1971 [Patrologia Orientalis, $36 / 2, \mathrm{n}^{\circ}$ 168], p. 294 [156] / 295 [157]; per l'ambito ambrosiano: Manuale Ambrosianum, II, pp. 195-197). Quest'ultimo aspetto era stato fedelmente continuato nella tradizione ambrosiana dall'età antica fino al 1983, quando fu pubblicato il II volume della Liturgia delle Ore riformata (Liturgia delle Ore secondo il Rito della Santa Chiesa Ambrosiana, II, Milano, 1983), in cui la celebrazione appare omologata allo schema romano: cfr. C. ALZATI, «Solemnitatum omnium honoranda solemnitas», p. 333-334.

${ }^{18}$ Cfr. C. ALZATI, Il salmo e l'incenso, p. 112-113.

19 Prima, Tertia, Sexta, Nona: BEROLDUS, p. 86-87; quanto al Completorium, esso appare semplicemente nominato (p. 47. 27-28). Nelle Domeniche (p. 47) e nelle festività (il Natale, ad esempio: p. 78), la Messa appare di norma preceduta da Terza; mentre nelle liturgie stazionali è il rituale stazionale ad introdurre alla celebrazione eucaristica. Significativamente nel Manuale di Valtravaglia (in cui si riflette la prassi dei collegi presbiterali in cura d'anime: urbani [decumani] e plebani) il normale ordo quotidiano registra semplicemente: Ad Matut. (con officiatuta notturna e Lodi matutine seguite dalla Messa) e Ad Vesperas; in Quaresima con l'aggiunta, Mane, relativa alla lettura di Genesi e Proverbi. 
no, risultano rintracciabili, almeno in parte, anche dopo le riforme seguite al concilio Vaticano $\mathrm{II}^{20}$, i documenti relativi alla celebrazione eucaristica affiancano, a una marcata continuità nell'ordinamento delle letture ${ }^{21}$, una evidente recezione del patrimonio eucologico romano, compresa la forma unitaria e invariabile della preghiera eucaristica. Peraltro, le ricerche di Matthieu Smyth ${ }^{22}$ hanno documentato negli antichi padri della provincia ecclesiastica milanese, quali il bresciano Gaudenzio e Massimo di Torino, la presenza di formule rituali successivamente riscontrabili in ambito gallicano e ispanico ${ }^{23}$.

Questo dato indica che le reliquie eucologiche di tipo 'gallicano' conservatesi nella celebrazioni eucaristiche «in coena Domini» e al culmine della Veglia pasquale, possono considerarsi le testimonianze residuali del patrimonio eu- cologico un tempo in uso nella Chiesa milanese: Matthieu Smyth ha parlato al riguardo di radicamento in un fondo molto antico condiviso con le Chiese delle Gallie e della Spagna, definibile «di tipo gallicano» nel senso di «non romano ${ }^{24}$; si tratta della tipologia di preghiera eucaristica scalzata dal formula eucologica fissa, impostasi nelle Chiese d'ambito suburbicario e a Roma ${ }^{25}$. La recezione di tale preghiera anche ad opera della Chiesa milanese non stupisce qualora si tengano presenti le vicende di tale Chiesa tra VI e VII secolo quando, dal 569 al 643 circa, il metropolita vicarius Ambrosii ${ }^{26}$ visse nella Liguria marittima sotto la tutela dell'autorità imperiale, per la quale l'arcivescovo di Ravenna e il papa romano costituivano i presuli di riferimento per le istituzioni ecclesiastiche in Italia ${ }^{27}$. Del resto, il Missale Bobiense, che risale probabilmente alla prima parte del secolo

Il manoscritto più antico dell'Ordo et caeremoniae beroldiano a noi pervenuto (Milano, Biblioteca Ambrosiana, ms. I 152 inf.) è collocabile attorno al 1140; per la confezione di tale codice: M. FERRARI, Valutazione paleografica del codice ambrosiano di Beroldo, in Il Duomo cuore e simbolo di Milano. IV Centenario della Dedicazione. 1577-1977, Milano, 1977 [Archivio Ambrosiano, 32], p. 302-307; per la composizione dell'Ordo, pochi anni prima, attorno al 1130: G. FORZATTI GOLIA, Le raccolte di Beroldo, Ibidem, p. 308-402; quanto alla nuova redazione del 1269, con integrazione dell'Ordo stesso all'interno di un Manuale: Ibidem, p. 330 ss. Sull'importanza dell'opera beroldiana, considerata nell'insieme delle sue componenti, cfr. anche P. CARMASSI, Libri liturgici e istituzioni ecclesiastiche a Milano in età medioevale. Studio sulla formazione del lezionario ambrosiano, Münster, 2001 (Liturgiewissenschaftliche Quellen und Forschungen, 85: Corpus ambrosiano-liturgicum, 4), p. 179 ss. La continuità tra alcune indicazioni cerimoniali beroldiane e le fonti non soltanto d'età carolingia, ma pure d'ascendenza più antica: Ibidem, p. 273-274.

Il rito 'ecclesiale' a Milano era di fatto il rito celebrato nelle «ecclesiae» del complesso arcivescovile o nelle stationes dall'arcivescovo assistito dai cardinali (o «ordinarii»), che potevano anche supplire all'assenza del presule. Oltre a essi il clero urbano annoverava i presbiteri decumani, incaricati della cura animarum. Per l'ordinamento del clero milanese in età medioevale e per l'articolazione dei ministeri previsti per gli ecclesiastici appartenenti agli ordini minori: E. CATTANEO, Istituzioni ecclesiastiche milanesi, in Storia di Milano, IV: Dalle lotte contro il Barbarossa al primo signore (1152-1310), Milano, 1954, p. 613-721; P. CARMASSI, Gli ordines della Chiesa milanese, in Libri liturgici e istituzioni ecclesiastiche a Milano (precedentemente cit.), p. $164-196$.

${ }^{20}$ Per una presentazione di queste ultime si rinvia al numero monografico de La Scuola Cattolica, 114 (1986), con contributi di G. TERRANEO (La liturgia delle ore ambrosiana: vicende, ragioni e prospettive di un cammino di riforma, p. 173-234), M. NAVONI (La nuova liturgia ambrosiana delle ore: struttura storico-comparativa, p. 235-324), C. MAGNOLI ("Un Direttorio non solo per la celebrazione ma anche per la meditazione". Confronto tra la "Institutio" ambrosiana delle ore e quella romana, p. 325-351).

${ }^{21}$ C. ALZATI, "Pollens ordo lectionum". Brevi note sulla storia del Lezionario Ambrosiano, in Il Lezionario secondo il Rito della Santa Chiesa di Milano (= Ambrosius, fasc. 1 [2009]: Numero speciale a cura della Congregazione del Rito Ambrosiano), p. 7-68.

${ }^{22}$ M. SMYTH, La liturgie oubliée. La prière eucharistique en Gaule antique et dans l'Occident non romain, Paris, 2003 (Patrimoines. Christianisme), p. 4547; e con particolare riferimento alle formule di embolismo al mandato eucaristico: p. 422-423.

${ }^{23}$ GAUDENTIUS Brixiensis, Tractatus II in Exodum, 31, in Opera, ed. A. Glück, Vindobonae-Lipsiae, 1936 (CSEL, 68), p. 31 [exemplar passionis Christi ante oculos habentes], con assonanze rispetto al Post Pridie [habentes ante oculos tantae passionis triumphos] del Liber Mozarabicus Sacramentorum, ed. M. Férotin (Paris, 1912), n 607, curr. A. Ward - C. Johnson, Roma, 1995 (Bibliotheca «Ephemerides Liturgicae». Subsidia, 78: Instrumenta Liturgica Quarreriensia, 4), p. 250; e del gallicano Sacramentario palinsesto di Milano: ed. A. Dold, Das Sakramentar im Schabcodex M 12 Sup. der Bibliotheca Ambrosiana. Mit hauptsächlich altspanischem Formelgut in gallischem Rahmenwerk, Beuron, 1936 (Texte und Arbeiten, 43), p. 30*. Cfr. anche l'embolismo al mandato eucaristico [usque quo iterum Christus de caelis adueniat] in riferimento alla formula [donec iterum adueniam] del De Sacramentis [IV, VI, 26: SCh, 25 bis, p. 116] e del Sacramentario irlandese palinsesto di Monaco [n ${ }^{\circ}$ 15, edd. A. Dold - L. Eizenhöfer, Das irische Palimpsestsakramentar im CLM 14429 der Staatsbibliothek München, Beuron 1964 (Texte und Arbeiten, 53-54), f. 10 v, p. 16]; nonché alla formula [donec ueniat in claritatem de celis] dei successivi libri ispanici [Missale mixtum: PL, 85, c. 553] e alla formula [donec iterum de caelis ueniam ad uos] del successivo Canone ambrosiano [si veda: Messale di Biasca, $n^{\circ}$ 768, ed. O. Heiming, cit. nota 35, p. 106-107]. Quanto a Massimo: Quotiescumque hoc feceritis, memoriam mei facietis, donec ueniam: MAXIMUS Taurinensis, Sermo XXXIX, 2, in Sermones, ed. A. Mutzenbecher, Turnholti, 1962 (CCL, 23), p. 152. A queste testimonianze può aggiungersi anche quella di un'altra omelia d'ambito «italiciano» del V secolo, designata come Sermo LXXVIII dello Pseudo Massimo: Quotiescunque haec feceritis, mortem meam annuntiabitis, donec ueniam: PL, 57, c. 690.

${ }^{24}$ M. SMYTH, La liturgie oubliée, p. 103.

${ }^{25}$ Sui caratteri strutturali della preghiera eucaristica romana, cfr. ultimamente E. MAZZA, Sul Canone della messa citato nel De Sacramentis di Ambrogio, in Ambrogio e la liturgia, cur. R. PASSARELLA, Milano-Roma, 2012 (Studia Ambrosiana, 6), p. 47-68.

${ }^{26}$ Tale è la definizione del metropolita milanese proposta nel GREGORII I Registrum, XI, 6, ed. D. Norberg, Turnholti, Brepols, 1982 (CCL, 140, A), p. 868. Cfr. C. ALZATI, Sancto Ambrosio servientes clerici. Una Chiesa, un presbiterio, l'ecumene, «La Scuola Cattolica», 134 (2006), p. 19-33.

${ }^{27}$ Già il secondo successore di Onorato, autore della migrazione a Genova, ossia il «patriarca» Lorenzo II, recatosi a Roma, sperimentò quasi contemporaneamente a Severo d'Aquileia la sottomissione alla condanna dei Tre Capitoli (GREGORII I Registrum, IV, 2, CCL, 140, p. 218), vissuta peraltro non senza ambiguità (GREGORII I Registrum, IV, 37, CCL, 140, p. 257). Per la qualifica di «patriarca», in un'accezione peraltro assolutamente generica non inusuale in ambito merovingico, attribuita a Lorenzo nel titolo redazionale di una lettera a lui indirizzata dal re franco Childeberto II attorno al 585: Epistolae Austrasicae, XLVI, ed. W. Gundlach, Berolini, 1892 (Monumenta Germaniae Historica [= MGH], Epistolae, III: Epistolae Merowingici et Karolini Aevi, I), p. 151. Con la elezione di Costanzo quale metropolita nel 593, il papa romano venne ad esercitare nella relativa procedura una decisiva e inusitata funzione, riservandosi l'assensus (GREGORII I Registrum: III: 29, 30 31, CCL, 140, p. 175-177; XI: 6, 14, CCL, 140, A, p. 867-868, 880) e conferendo al nuovo metropolita il pallio petrino (GREGORII I Registrum, IV, 1, CCL, 140, p. 218). Tale 'presenza' del papa romano si sarebbe immediatamente evidenziata anche nella comune vita istituzionale della provincia, talvolta attraverso il supporto offerto alle decisioni del metropolita (GREGORII I Registrum, IX, 224, CCL, 140, A, p. 798), ma in altre occasioni impartendo a lui precise direttive cui attenersi (GREGORII I Registrum: V, 18, CCL, 140, p. 285-287; IX: 187, 235, CC, 140, A, p. 743-744, 818-819). La stessa ordinazione episcopale dell'italico Birino, destinato quale missionario nel Wessex, commissionata attorno al 634 da papa Onorio al milanese Asterio in Genova, s'inserisce in tale logica (BEDA, Historia Ecclesiastica Gentis Anglorum, III, 7, 1, ed. M. Lapidge, Paris, 2005 [SCh, 
VIII e la cui origine resta incerta, attesta anche in ambito gallicano un'analoga recezione del Canon actionis romano e della conseguente struttura celebrativa ${ }^{28}$.

Quanto al più generale influsso dell'eucologia romana, fenomeno comune a tutto l'Occidente ${ }^{29}$, in ambito milanese esso èverificabile già nel frammento di Libellus Missarum del secolo VII, presente nel codice palinsesto 908 di San Gallo ${ }^{30}$. Poiché la presente indagine intende focalizzare l'attenzione in particolare sui libri ambrosiani per la celebrazione eucaristica in ambito monastico, va subito osservato che le fonti codicologiche sono rappresentate da manoscritti, la cui elaborazione si lega strettamente al periodo carolingio e alla cultura ecclesiastica, che lo caratterizzò nei domini franchi, tra i quali dopo il 774 anche Milano si trovò inserita. Va rimarcato come proprio in tale periodo la tradizione ambrosiana della Chiesa milanese abbia conosciuto un poderoso rilancio, traducendosi in creazioni letterarie e artistiche, che avrebbero continuato a ispirare per secoli la scientia Ambrosiana ${ }^{3}$.

Nel 1974, ad opera della badessa Judith Frei, apparve l'edizione di un testo particolarmente rilevante in rapporto alle questioni di cui ci stiamo occupando. Si tratta del messale del monastero urbano di San Simpliciano ${ }^{32}$. Nell'Einleitung a tale edizione, la Frei - oltre ad alcune importanti consi-

39o], p. 44). Per i legami di Genova con l'area inglese in quel periodo, cfr. U. FORMENTINI, Genova nel Basso Impero e nell'Alto Medioevo, in Storia di Genova dalle origini al tempo nostro, Milano 1941, p. 110-111). Sotto Costanzo un segnale assai eloquente dell'indebolita situazione del metropolita milanese fu l'instaurarsi della prassi di far memoria nelle proprie celebrazioni dell'arcivescovo di Ravenna, sede del rappresentante del potere imperiale: un uso che peraltro venne lasciato rapidamente cadere, su consiglio dello stesso papa Gregorio, per l'indignata reazione dei comprovinciali (GREGORII I Registrum, IV, 37, CCL, 140, p. 258).

${ }^{28}$ Ed. E. A. Lowe, The Bobbio Missal: A Gallican Mass-Book (Ms. Paris Lat. 13246), London, 1920-1924 (Henry Bradshaw Society, 58-59).

${ }_{29}$ Valore esemplare assume al riguardo il cosiddetto Missale Gallicanum Vetus [s. VII ${ }^{\text {ex. }}$-VIII ${ }^{\text {in. }}$ ], che - in una salvaguardata struttura di tipo gallicano - massicciamente inserisce formule eucologiche romane: Missale Gallicanum Vetus (Cod. Vat. Palat. Lat. 493), edd. L. C. Mohlberg - L. Eizenhöfer - P. Siffrin, Roma, 1958 (Rerum Ecclesiasticarum Documenta. Series Maior. Fontes, 3).

$3^{30}$ A. DOLD, Le text de la Missa catechumenorum du Cod. Sangall. 908, in Revue Bénédictine, 36 (1924), p. 307-316; Id., Getilgte Paulus- und Psalmentexte unter getilgten ambrosianischen Liturgiestücken aus Codex Sangall. 908, Beuron, 1928 (Texte und Arbeiten, 14); Id., Dove si trovano le più antiche tracce della Messa milanese, in Quaderni di Ambrosius [Supplemento ad Ambrosius, 37 (1961)], p. 3-15. Il significato di questo testimone per la storia della liturgia milanese è stato recentemente posto in luce da P. CARMASSI con nuove considerazioni nel già citato volume (si veda nota 19) Libri liturgici e istituzioni ecclesiastiche a Milano, p. 106-130: Il libello palinsesto del VII secolo nel codice St. Gallen, Stiftsbibliothek, 908.

${ }^{31}$ Sulla scientia Ambrosiana e sul luogo della sua trasmissione, ossia le scuole annesse alla ecclesia beatae Mariae, quae huius archiepiscopatus ... caput extitit et Deo annuente semper existet: L(ANDULFUS), Historia Mediolanensis, II, 35, edd. L. C. Bethmann - W. Wattenbach, Hannoverae, 1848 (MGH, Scriptores, VIII), p. 70 ss.; cfr. - con carenze critiche, ma migliore base testuale - ed. A. Cutolo, Bologna, 1942 (Rerum Italicarum Scriptores, editio altera, IV, 2), p. 75 ss. Quanto al nome dell'autore di questa narrazione medioevale, la tradizione manoscritta trasmette esclusivamente la lettera iniziale: cfr. J. W. BUSCH, "Landulfi senioris Historia Mediolanensis" - Überlieferung, Datierung und Intention, «Deutsches Archiv», 45 (1989), p. 11-12. In merito alla datazione, il Busch, distinguendo dal resto dell'opera gli ultimi (e per lui successivi) quattro capitoli, propende per un anno di composizione non lontano dal 1075; un quadro della questione anche in P. CHIESA, Landolfo Seniore, in Dizionario Biografico degli Italiani, 63, Roma, 2004, pp. 498-499. La datazione di Jörg Busch è stata pacificamente assunta dalla storiografia tedesca: cfr. Ch. DARTMANN, Wunder als Argumente: die Wunderberichte in der Historia Mediolenenesis des sogenannten Landulf Senior und in der Vita Arialdi des Andrea von Strumi, Frankfurt am Main, 2000 (Gesellschaft, Kultur und Schrift, 10), si veda in particolare nota 168, pp. 120-121; O. ZUMHAGEN, Religiöse Konflikte und kommunale Entwicklung: Mailand, Cremona, Piacenza und Florenz zur Zeit der Pataria, Köln, 2002 (Städteforschung, 58), p. 29. Personalmente, considerando lo scritto, quale ci è pervenuto, una raccolta di testi, in gran parte di scuola, ed escludendo le interpolazioni successive ipotizzate dal Busch a giustificazione della propria datazione precoce, propendo per una redazione tarda (primissimi anni del secolo XII; cfr. già W. WATTENBACH, Deutschlands Geschichtsquellen im Mittelalter bis zur Mitte des dreizehnten Jahrhunderts, II, Berlin, $1894^{6}$, p. 242): C. ALZATI, Chiesa ambrosiana, mondo cristiano greco e spedizione in Oriente (in Verso Gerusalemme, II Convegno internazionale nel IX Centenario della I Crociata [1099-1999]. Bari, 11-13 gennaio 1999), «Civiltà Ambrosiana», 17 (2000), p. 32-35, 40-41, 44-45; ID., Parlare con la voce dei Padri. L'apologetica ambrosiana di fronte ai riformatori del secolo XI, in Leggere i Padri tra passato e presente, Cremona, 21-22 Novembre 2008, Cur. M. Cortesi, Firenze 2010, nota 27; a conclusioni cronologiche non dissimili sembra giungere, seppure per altra via, anche P. CARMASSI, Basiliche episcopali e ordinamento liturgico a Milano nei secoli XI-XIII tra continuità e trasformazioni, «Civiltà Ambrosiana», 17 (2000), pp. $268-291$.

La ecclesia di Santa Maria, detta anche Santa Maria Maggiore, era la ecclesia hiemalis, che Arnolfo nell'ultimo quarto dell'XI secolo appella Sancta Theotocos (ARNULFUS, Liber gestorum recentium, ed. I. Scaravelli, Bologna, 1996 [Fonti per la storia dell'Italia medievale ad uso delle scuole, 1], I, 19, p. 78. 24-25).

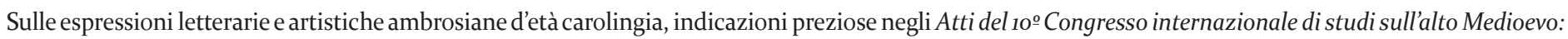
Milano e i milanesi prima del Mille, Milano, 26-30 settembre 1983, Spoleto, 1986. Tra le composizioni di carattere agiografico di quel periodo spicca il De vita et meritis Ambrosii, ed. P. Courcelle, Recherches sur saint Ambroise. Vies anciennes, culture, iconographie, Paris, 1973 (Études Augustiniennes, Antiquité, 52). Per la datazione agli anni dell'arcivescovo Angilberto II (824-860): P. TOMEA, Ambrogio e i suoi fratelli. Note di Agiografia milanese altomedioevale, «Filologia mediolatina», 5 (1998), p. 149-232, che ha ripreso e ulteriormente documentato un'ipotesi affacciata già da L. CRACCO RUGGINI (recensione a Vita e meriti di s. Ambrogio, cur. A. PAREDI, Milano, 1964 [Fontes Ambrosiani, 37], «Athenaeum», n. s., 43 [1965], p. 237, 239). Allo stesso TOMEA si deve la convincente collocazione del De situ civitatis Mediolani agli anni iniziali dell'XI secolo, segnati dalla figura dell'arcivescovo Arnolfo II: Tradizione apostolica e coscienza cittadina a Milano nel medioevo. La leggenda di san Barnaba, Milano, 1993 (Bibliotheca erudita, 2), p. 418 ss.

${ }_{32}$ Das ambrosianische Sakramentar D 3-3 aus dem mailändischen Metropolitankapitel, ed. J. Frei, Münster, 1974 (Liturgiewissenschaftliche Quellen und Forschungen, 56: Corpus Ambrosiano-liturgicum, 3). Quanto alla datazione, la Frei propendeva per la conclusione del IX o l'avvio del X secolo: Ibidem, Einleitung, p. 19-21. A. PAREDI aveva a suo tempo assegnato il ms. al secolo XI (Messali ambrosiani antichi, in Quaderni di Ambrosius [Supplemento ad Ambrosius, 35 (1959)], p. 16]; successivamente A. M. TRIACCA, Libri liturgici ambrosiani, in Anamnesis. 2. La Liturgia. Panorama storico generale, dir. S. Marsili, Casale, 1978, p. 206, parlò di secolo IX; alla seconda metà del secolo IX lo assegnò M. FERRARI nel contributo su Manoscritti e cultura, all'interno degli Atti del $10^{\circ}$ Congresso del Centro spoletino (volume citato al termine della precedente nota), p. 263. Nei suoi Codices Liturgici Latini Antiquiores (Freiburg Schweiz, $1968^{2}$ [Spicilegii Friburgensis Subsidia, 1], I, $n^{\circ}$ 510, p. 265-266), Klaus GAMBER aveva parlato di fine IX secolo, ma nel Supplementum A. M. TRIACCA (Freiburg Schweiz, 1988 [Spicilegii Friburgensis Subsidia, 1, A], p. 63) ha fatto propria la proposta di E. B. GARRISON (Random Notes on Early Italian Manuscripts, in La Bibliofilia, 8o [1978], p. 211, $\mathrm{n}^{\circ} 2$ [ried. in Early Italian Painting. Selected Studies, 2, London, 1984, p. 323, $\mathrm{n}^{\circ}$ 2]), per una datazione al secolo X-XI. Come strettamente dipendente dal Messale di S. Simpliciano è stato valutato il palinsesto London, British Library, Harleian 2510, analizzato da O. HEIMING, Ein «fusioniertes» Gregorianum und ein Ambrosiano-Benedictinum. Zwei Palimpsest-Sakramentare im British Museum Harleian 2510, in Ephemerides Liturgiacae, 64 (1950), p. 238-273. Al riguardo, cfr. anche J. FREI, in Das ambrosianische Sakramentar D 3-3, p. 6, nº 10; p. 119, nota 404. 
derazioni in merito alla struttura degli antichi formulari eucaristici milanesi33 - ipotizzava la precocità di quanto documentato dal codice di San Simpliciano rispetto alla tradizione testuale prevalente negli altri messali d'ambito ambrosiano $^{34}$ (e segnatamente rispetto al Messale di Biasca) ${ }^{35}$, tradizione testuale, che altre fonti medioevali documentano concordemente come radicata nelle ecclesiae del complesso arcivescovile e nella prassi celebrativa del clero cardinalizio, che in tali chiese - con o senza arcivescovo - officiava ${ }^{36}$.

Che il manoscritto del monastero di San Simpliciano avesse all'origine un libro rituale esemplato sulle celebrazioni delle ecclesiae del complesso arcivescovile è reso evidente dall'indicazione archiepiscopus per designare l'officiante nella celebrazione del Sabato Santo ${ }^{37}$; che inoltre costituisse una declinazione di quel modello per un ambito monastico trova riscontro nella sistematica eliminazione di tutte le celebrazioni a Pasqua e nell'Ottava pasquale legate ai riti di iniziazione cristiana ${ }^{3}$.

La collocazione di tale testo nel processo evolutivo del messale in ambito ambrosiano è stata notevolmente modificata dall'edizione nel 2003, ad opera di Gabriele Verità, del Messale di Armio (ms. II D 3. 1, della Biblioteca del Capitolo metropolitano di Milano) ${ }^{39}$. In effetti, quest'ultimo messale, denominato dalla località della Val Veddasca, nella cui chiesa di San Lorenzo fu conservato per secoli, è risultato essere testimone di una redazione di messale ad uso monastico più arcaica rispetto a quella del Messale di San Simpliciano e anche strutturalmente più prossima ai modelli legati alle ecclesiae del complesso episcopale ${ }^{40}$ : una redazione che San Simpliciano presuppone e rielabora, integrando l'eucologia di origine gelasiana con testi tratti da fonti gregoriane ${ }^{41}$.

In questa redazione monastica del messale ambrosiano, particolarmente significativo appare l'ordinamento sia delle letture per le ferie di Quaresima (attestate in San Simpliciano, ma solo parzialmente e indirettamente in Armio, stante la lacuna iniziale), sia di quelle per le Domeniche dopo Pentecoste. Va osservato che le ferie di Quaresima nelle celebrazioni cardinalizie nella ecclesia hyemalis prevedevano, con evidente radicamento nell'età patristica, la lectio continua di Genesi e Proverbi, seguita dalla proclamazione del Sermone del Monte, pure in lectio continua, dal Vangelo secondo Matteo. Dovendo approntare un libro unitario per la celebrazione al di fuori delle solenni cerimonie di Santa Maria Maggiore, la redazione del messale conservò le pericopi evangeliche ${ }^{42}$, ma pose quale lectio i testi stabilitisi in ambito romano ${ }^{43}$. San Simpliciano testimonia la scelta di mutuare dall'ambito romano l'intero ordinamento ${ }^{44}$, ma due rimandi interni del Messale di Armio suggeriscono che tale scelta già dovette essere nell'archetipo da cui Armio stesso era stato tratto45.

Quanto alle Domeniche dopo Pentecoste, nel cui ciclo spiccavano le due feste della Decollazione e della Dedicazione, in età precarolingia a Milano sono attestate pericopi in lettura progressiva dai Vangeli di Matteo, Marco e Luca ${ }^{46}$. Furono i redattori d'età carolingia a riformulare in modo organico l'intero ciclo, assegnando pericopi proprie ad ogni Domenica. Come per la Quaresima i messali monastici scelsero invece di mutuare totalmente le serie di letture dai testi romani. Si trattava con ogni evidenza di materiale con cui i redattori avevano particolare consuetudine (dato quanto mai ovvio, trattandosi di monaci, che - pur nella stabilitas loci - partecipavano della molteplicità di relazioni e scambi intellettuali, di cui il monachesimo dà testimonianza in età carolingia). Del resto, il fatto che i fruitori di tali messali potessero non appartenere originariamente alla tradizione rituale ambrosiana è suggerito anche da un'ulteriore interessante particolarità del Messale di San Simpliciano. In ambito ecclesiale ambrosiano, nei messali, sono ovviamente omessi i dialoghi rituali, la cui conoscenza era data per scontata. Ma in San Simpliciano il redattore ritenne opportuno (o, forse,

\footnotetext{
33 J. FREI, Einleitung, in Das ambrosianische Sakramentar D 3-3, p. 141 ss.

${ }^{34}$ Un'accurata presentazione del lavoro della Frei in B. (G.) BAROFFIO, Verso una storia dell'antica eucologia ambrosiana: Judith Frei e l'edizione del Sacramentario di S. Simpliciano, in Ricerche storiche sulla Chiesa ambrosiana, VII, Milano, 1977 (Archivio Ambrosiano, 33), p. 5-25.

${ }_{35}$ Das ambrosianische Sakramentar von Biasca: die Handschrift Mailand Ambrosiana A 24 bis inf., I, ed. O. Heiming, Münster, 1969 (Liturgiewissenschaftliche Quellen und Forschungen, 51: Corpus Ambrosiano-liturgicum, 2).

${ }^{36}$ Preziosa documentazione al riguardo offre il già citato BEROLDUS (vedasi sopra, note 17, 19).

${ }_{37}$ Das ambrosianische Sakramentar D 3-3, p. 316. 5.

$3^{8} \mathrm{Ibidem}$, p. 216 ss.

39 G. VERITÀ, Il Messale di Armio. Edizione e commento, in Ricerche Storiche sulla Chiesa Ambrosiana, XXI, Milano, 2003 (Archivio Ambrosiano, 88), p. 5-197. Il manoscritto purtroppo si presenta mutilo della prima sezione fino alla Feria VI in Albis. Lo stesso VERITÀ, alle p. 17-18, ricorda che A. PAREDI (Messali ambrosiani antichi, p. 18) aveva datato il codice al s. XI, e che su tale valutazione aveva concordato anche R. AMIET (La tradition manuscrite du missel ambrosien, «Scriptorium», 14 [196o], p. 32). Il Paredi era poi tornato sulla questione, ripiegando sul secolo X (Sacramentariium Bergomense. Manoscritto del secolo IX della Biblioteca di S. Alessandro in Colonna di Bergamo, Bergamo, Monumenta Bergomensia, 1962, p. XII, nota 6). Quest'ultima datazione fu condivisa anche da K. GAMBER (Codices Liturgici Latini Antiquiores, I, ${ }^{\circ}{ }^{518}$ ), ma nel Supplementum, A. M. TRIACCA, recependo le considerazioni di E. B. GARRISON (Random Notes, p. $211, \mathrm{n}^{\circ} 3$ [p. 323, $\mathrm{n}^{\circ} 3$ ]), ha riproposto la collocazione al s. XI. J. FREI (Einleitung, p. 5, nota 25), si è attenuta alla datazione comunicatale da Bernard Bischoff: fine del IX, o comunque tra IX e X secolo; è la datazione recepita anche da M. Ferrari nella scheda interna alla Biblioteca del Capitolo metropolitano milanese, nell'aggiornamento del 1995.

$4^{\circ}$ Cfr. G. VERITÀ, Il Messale di Armio, p. 18-21.

${ }^{41}$ Ibidem, p. 73 ss.

${ }^{42}$ Cfr. Das ambrosianische Sakramentar von Biasca, ed. O. Heiming, p. 38 ss.

${ }^{43}$ Ibidem.

${ }_{44}^{4}$ Das ambrosianische Sakramentar D 3-3, p. 174 ss.

${ }^{45}$ Cfr. G. VERITÀ, Il Messale di Armio, p. 25-26.

${ }^{46}$ A. PAREDI, L'Evangeliario di Busto Arsizio, in Miscellanea Liturgica in onore di Sua Eminenza il cardinale Giacomo Lercaro, II, Roma-Parigi-Tournai-New York, 1967, p. 246-248. Per la documentazione relativa alle evoluzioni determinatesi in età carolingia, si vedano le tabelle elaborate da P. CARMASSI, Appendice: Le letture per le Domeniche dopo Pentecoste, in Libri liturgici e istituzioni ecclesiastiche a Milano (cit. nota 19), p. 124-13o.
} 
necessario) riportarli puntualmente (un aspetto che, tra l'altro, si è rivelato preziosissimo anche per gli studiosi) ${ }^{47}$.

\section{LA DECLINAZIONE MONASTICA DEL RITO AMBROSIANO FUORI MILANO: LA PRAGA DI CARLO IV}

Ma il rito ambrosiano monastico non fu esperienza limitata al solo ambito urbano milanese. In occasione dell'Epifania del 1355 nella Basilica Ambrosiana, il rex Romanorum (dal 1349) Carlo, figlio del re di Boemia Giovanni di Lussemburgo e di Eliška (erede diretta della dinastia Přemyslide, che gli trasmise il nome di Václav [Venceslao]), ricevette la corona ferrea del Regnum Italiae quale premessa all'incoronazione imperiale svoltasi nella Pasqua di quello stesso anno a Roma ${ }^{48}$.

Se già quale margravio di Moravia e governatore di Boemia egli aveva dedicato un'amorosa attenzione alla città di Praga, dopo l'acquisizione del trono boemo la cura per l'immagine di questa sua sede avrebbe portato ad esiti grandiosi, che permisero alla Praga carolina di rivaleggiare in magnificenza con Roma e Costantinopoli ${ }^{49}$. Tale splendore rifletteva la percezione, che Carlo ebbe, della propria dignità istituzionale e il vasto orizzonte entro cui egli concepì la propria azione di governo.

Era ancora "margravio di Moravia", e sovrintendeva alla Boemia sotto l'autorità del padre, quando dalla Croazia chiamò nella Nové Město una comunità benedettina, per dare vita sulle rive della Vltava a un cenobio di rito glagolitico $^{50}$. Già la seconda generazione di monaci fu composta da cechi che «scrissero in lingua ceca nella scrittura glagolitica ${ }^{51}$. La fondazione fu originariamente concepita come iniziativa volta ad esprimere la fraternità e a favorire l'unità slava, secondo la prospettiva che pure la successiva lettera allo car' Stefano Dušan avrebbe manifestato e che la storiografia ceca non ha mancato di rimarcare ${ }^{52}$. Tuttavia quando, ormai era divenuto rex Romanorum, Carlo diede vita a un'ulteriore singolare fondazione, la cui vicenda venne di fatto intrecciandosi a quella del cenobio ad Sclavos (na Slovanech), non senza riflessi per la stessa immagine ideale di quest'ultimo.

Per volontà imperiale, preparata da una previa concessione papale del 26 febbraio $1353^{53}$, sorse infatti nell'estremo settentrione di Nové Město, presso la Porta delle Polveri (Prašná Brána), una nuova abbazia dedicata a sant'Ambrogio, nella quale i monaci, provenienti dal cenobio milanese annesso alla Basilica Ambrosiana, dovevano «missas et alia divina officia secundum ritum Ambrosianum legere et cantare ${ }^{54}$. Un'ulteriore lettera pontificia del 28 dicembre 1359 ci attesta come nella visione di Carlo, ormai a tutti gli effetti Romanorum imperator semper augustus, le due istituzioni fossero venute assimilandosi nel loro significato ideale. Papa Innocenzo VI infatti, in conformità al volere dell'imperatore, accordò che in presenza dell'imperatore stesso gli abati e i monaci dei detti monasteri potessero, in qualunque luogo si trovassero, celebrare secondo i rispettivi riti ${ }^{55}$.

Strettamente collegata alla persona dell'augusto, la varietà rituale diveniva così segno dell'estensione ecumenica della di lui autorità ed esprimeva quell'unità, ad un tempo multiforme e armonica, che - nella lettura del nuovo Carlo - costituiva la nota specifica dell'Impero. A questa presenza ambrosiana nella Praga imperiale mise violentemente fine la rivoluzione hussita ${ }^{56}$.

Merita osservare come, pure nel contesto praghese, il carattere monastico della comunità abbia comportato specifiche implicazioni per l'ordinamento rituale: mentre le celebrazioni diurne, compresa la celebrazione eucaristica, si

\footnotetext{
$\overline{47}$ Das ambrosianische Sakramentar D 3-3, p. 268 ss.; cfr. Einleitung, p. 74 ss.

${ }^{48}$ Per l'incoronazione e il soggiorno milanese: Die Regesten des Kaiserreichs unter Kaiser Karl IV.: 1346-1378, post J. F. Böhmer ed. A. Huber, Innsbruck, 1877 (ried. an., Hildesheim, 1968) (Regesta Imperii, VIII), nn. 1962 b - 1971 a. Cfr. R. PASTĖ, L'incoronazione di Carlo IV a Milano, in La Scuola Cattolica, 62 (1934), p. 339-344. Per Roma: Die Regesten des Kaiserreichs, nn. 2013 a - 2059. La corona ferrea usata per Carlo era quella già approntata per il nonno Arrigo VII nel 1311 e da questi affidata all'abate di Sant'Ambrogio (cfr. R. ELZE, Die “Eiserne Krone” in Monza, in P. E. SCHRAMM, Herrschaftszeichen und Staatssymbolik, II, Stuttgart, 1955 [MGH, Schriften, XIII, 2], p. 475). Cfr. anche R. ELZE, La corona imperiale di paglia, in Cultura e società nell'Italia medievale. Studi per Paolo Brezzi, I, Roma 1988 (Studi Storici, 184-192), p. 337-344; sugli ordines milanesi per l'incoronazione regia, di cui già s'era fruttuosamente occupato G. GIULINI, Memorie spettanti alla storia, al governo e alla descrizione della città e campagna di Milano, voll. 9+3, Milano, 1760-1765; 1771-1774

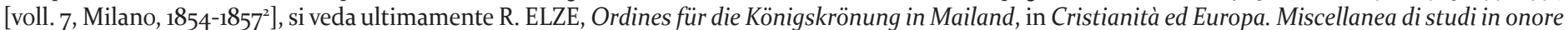
di Luigi Prosdocimi, I, 1, cur. C. Alzati, Roma-Freiburg-Wien, 1994, p. 175-189.

${ }_{49}$ Per la fase di governo anteriore all'incoronazione imperiale, fase in cui si ebbero, tra l'altro, l'elevazione della sede episcopale praghese al rango arcivescovile-metropolitico (1344) (dignità cui si sarebbe poi aggiunta la legazia apostolica), la ricostruzione del castello e della cattedrale di San Vito, la ricostruzione del castello di Vyšehrad, la fondazione della «Città Nuova» (Nové Město), l'istituzione dell’Università (1348): J. ŠUSTA, Karel IV., 2 voll., Praha, 1946-1948 (České Dějiny, II, 3-4). Più in generale, cfr. F. SEIBT, Karl IV., in Handbuch der Geschichte der Böhmischen Länder, cur. K. Bosl, Stuttgart, 1967, p. 348 ss.; F. DVORNÍK, Les Slaves, Paris, 1970, p. 363 ss.

$5^{50}$ L'approvazione papale alla nuova fondazione, ubicata nell'estremo settore meridionale della «Città Nuova», fu accordata nel 1346: Regesta Diplomatica nec non Epistolaria Bohemiae et Moraviae, IV: 1333-1346, ed. J. Emler, Pragae, 1892, nº 1704, p. 684. Dedicato originariamente al dalmata san Girolamo, dichiarato senz'altro natione Slavus, il monastero, in seguito alla dedicazione della chiesa il Lunedì di Pasqua del 1372, fu tradizionalmente designato e comunemente conosciuto col nome di «Emmaus». Per un accostamento delle più antiche testimonianze del rito glagolitico, si potrà vedere anche la ricca introduzione di SANTE GRACIOTTI a Il Messale Croato-Raguseo (Neofiti 55) della Biblioteca Apostolica Vaticana, post C. Giannelli (†) ed. S. Graciotti, Città del Vaticano, 2003 (Studi e Testi, 411).

${ }^{51}$ F. V. MAREŠ, Le origini liturgiche della lingua e della cultura nazionale degli Slavi, in Storia religiosa dei popoli balcanici, cur. L. Vaccaro, Milano-Gazzada, 1983 , p. 150.

${ }^{52}$ F. DVORNÍK, Les Slaves, p. 428, 481. Le concomitanti lettere pontificie al monarca serbo in Vetera monumenta Slavorum meridionalium historiam illustrantia, ed. A. Theiner, II, Romae, 1863, p. 8-17.

${ }^{53}$ Monumenta Vaticana res gestas Bohemicas illustrantia, II: Acta Innocentii VI. 1352-1362, ed. J. F. Novák, Pragae, 1907, n 19, p. 9-10.

${ }_{54}$ Per le prerogative dell'abate, gratificato dall'uso di mitra, anello, pastorale e delle altre insegne pontificali: $I b i d e m, n^{\circ} 21, p .10$.

55 «...quod dicti abbates et monachi dictorum monasteriorum et eorum singuli in quocumque loco ad hoc ydoneo extra sua monasteria possint in presencia dicti imperatoris Missas et alia divina officia secundum ritum eorum licite celebrare»: Ibidem, ${ }^{\circ}$ 1018, p. 406-407; il privilegio papale: Ibidem, ${ }^{\circ} 1019$, p. 407. ${ }^{56}$ Cfr. C. ALZATI, Dalla Slavia all'ecumene. Glagoliti e Ambrosiani nella Praga di Carlo IV, in Umanità e nazioni nel diritto e nella spiritualità da Roma a Costantinopoli a Mosca, XII Seminario Internazionale di Studi Storici “Da Roma alla Terza Roma” (Campidoglio, 21-23 aprile 1992), Roma, 1994, p. 73-84.
} 
svolgevano secondo il rito "ecclesiale" ambrosiano, l'officiatura antelucana assumeva un proprio andamento conforme al cursus monastico ${ }^{57}$. È curioso questo riproporsi - presso gli ambrosiani sulle rive della Vltava - di una modalità d'integrazione tra rito della ecclesia e rito dei monaci, che appare non molto dissimile da come tali distinte forme rituali erano venute armonicamente intrecciandosi nella vita cultuale dell'Anastasis gerosolimitana, descrittaci alla fine del IV secolo da Egeria ${ }^{5}$. L'integrazione, fin qui documentata, tra rito della ecclesia locale e rito delle locali comunità monastiche, fu fenomeno che a Milano sarebbe venuto meno con l'apparire degli Ordini mendicanti.

Sappiamo come l'unità rituale delle provincie ecclesiastiche sia stata un principio costantemente riaffermato nelle sinodi occidentali d'età tardo antica ${ }^{59}$, ma sia anche principio che la politica ecclesiastica del re franco Carlo ha violentemente contraddetto, con conseguenze irreversibili per la vita delle Chiese ${ }^{60}$. Sta di fatto che l'anomalia canonica instaurata da Carlo nella provincia ecclesiastica milanese, attraverso l'imposizione della tradizione rituale romana alle sedi suffraganee, ha finito per configurare come bizzarra anomalia l'originaria tradizione rituale ambrosiana, ininterrottamente perpetuatasi nella sede arcivescovile. In modo non dissimile, il nesso tra vita cultuale della ecclesia e forme rituali monastiche (che ha caratterizzato per secoli l'esperienza delle Chiese cristiane, compresa la Chiesa ambrosiana), venendo meno con l'affermarsi dei Mendicanti, ha determinato l'interrompersi di un'organica interazione tra le componenti della Chiesa locale, segno eloquente dell'unitaria vitalità del corpo ecclesiale.

57 «Fiat ita tamen quod abbas et conventus predicti duodecim lecciones et duodecim responsoria et duodecim psalmos cum tribus canticis una cum evangelio secundum morem ordinis s. Benedicti dicere teneantur»: Ibidem, nº 19, p. 10.

${ }^{58} \mathrm{~A}$ Gerusalemme, nelle ferie, era propria dei monaci (i monazontes) la preghiera salmica antelucana, cui seguiva la solenne officiatura mattutina celebrata dal vescovo e dal clero: «Singulis diebus ante pullorum cantum aperiuntur omnia hostia Anastasis et descendent omnes monazontes et parthene, ut hic dicunt, et non solum hii, sed et laici preter, uiri aut mulieres, qui tamen uolunt maturius uigilare. Et ex ea hora usque in luce dicuntur ymni et psalmi responduntur, similiter et antiphonae, et cata singulos ymnos fit oratio ... Iam autem ubi ceperit lucescere, tunc incipiunt matutinos ymnos dicere. Ecce et superuenit episcopus cum clero et statim ingreditur intro spelunca et de intro cancellos primum dicet orationem pro omnibus; commemorat etiam ipse nomina, quorum uult, sic benedicet cathecuminos. Item dicet orationem et benedicet fideles. Et post hoc exeunte episcopo de intro cancellos omnes ad manum ei accedunt, et ille eos uno et uno benedicet exiens iam, ac sic fit missa [ossia: dimissio] iam luce» (EGERIA, Itinerarium, XXIV, 1-2, SCh, 296, p. 234-236).

${ }_{59}$ Così si espresse, già tra il 416 e il 418, una sinodo bizacena riproposta nella prima metà del VI secolo dalla Breviatio canonum di Ferrando (Vt una sit in sacramentis per omne Byzacium disciplina [220]: Concilia Africae. A. 345 - A. 525, ed. Ch. Munier, Turnholti, 1974 [CCL, 149], pp. XXXVII, 305); analogamente si pronunciò nelle Gallie la sinodo di Vannes tra il 461 e il 491 (Vt uel intra prouinciam nostram sacrorum ordo et psallendi una sit consuetudo [can. 15]: Concilia Galliae. A. 314 - A. 506, ed. Ch. Munier, Turnholti, 1963 [CCL, 148], p. 155), e - con esplicito riferimento al modello autoritativo della sede metropolitana - quella di Yenne del 517 (Ad celebranda divina officia ordenem, quem metropolitani tenent, provincialis eorum observare debebunt [can. 27]: Concilia Galliae. A. 511 - A. 695, ed. Ch. de Clercq, Turnholti, 1963 [CCL, 148, A], p. 30). In quello stesso 517 in modo consonante avrebbe deliberato la sinodo tarraconense di Gerona (De institutione missarum, ut quomodo in metropolitana ecclesia fiunt, ita in Dei nomine in omne Terraconense prouincia tam ipsius missce ordo quam psallendi uel ministrandi consuetudo seruetur [can. 1]: Concilios Visigóticos e Hispano-Romanos, ed. J. Vives, p. 39). Ancora nel 633 il principio dell'unità rituale delle province ecclesiastiche sarebbe stato confermato dalla IV sinodo Toletana, resa particolarmente autorevole dalla prestigiosa presenza di Isidoro (Hoc enim et antiqui canones decreuerunt ut unaquaeque prouincia et psallendi et ministrandi parem consuetudinem teneat [can. 2]: Ibidem, p. 188). Cfr. CARMASSI, Libri liturgici e istituzioni ecclesiastiche a Milano, pp. 34-35; in tale denso volume la studiosa ha altresì verificato, attraverso un'accurata analisi della documentazione manoscritta superstite, gli aspetti di convergenza concretamente reperibili nell'ambito dell'antica provincia milanese.

${ }^{60}$ Singolare il compiacimento al riguardo di C. VOGEL, Les motifs de la romanisation du culte sous Pépin le Bref (751-768) et Charlemagne (774-814), in Culto cristiano e politica imperiale carolingia, Todi 1979 (Convegni del centro di studi sulla spiritualità medievale, XVIII: 9-12 ottobre 1977), pp. 15-41; cfr. dello stesso: Les échanges liturgiques entre Rome et les pays francs jusqu'à l'époque de Charlemagne, in: Le Chiese nei regni dell'Europa Occidentale e i loro rapporti con Roma sino all'8oo, Atti del convegno internazionale di studio (Spoleto, 7-13 aprile 1959), Spoleto, 196o (Settimane di studio sull'alto medioevo, 7), pp. 185-295. Nel secondo quarto del IX secolo Valafrido Strabone aveva ben presente l'esito di tale politica ecclesiastica dei monarchi carolingi in rapporto alla provincia ecclesiastica milanese: «Ambrosius quoque Mediolanensis episcopus tam missae, quam ceterorum dispositionem officiorum suae ecclesiae et alis Liguribus ordinavit, quae et usque hodie in Mediolanensi tenentur ecclesia»: WALAFRIDUS STRABO, Libellus de exordiis et incrementis quarundam in observationibus ecclesiasticis rerum, 23, ed. V. Krause, Hannoverae, 1897 (MGH, Leges, sect. II: Capitularia, II), p. 497. 11-13. 